Proceedings of the 2010 Winter Simulation Conference

B. Johansson, S. Jain, J. Montoya-Torres, J. Hugan, and E. Yücesan, eds.

\title{
PLANNING FOR INFECTIOUS DISEASE OUTBREAKS: A GEOGRAPHIC DISEASE SPREAD, CLINIC LOCATION, AND RESOURCE ALLOCATION SIMULATION
}

\author{
Sean Carr \\ Industrial and Systems Engineering \\ North Carolina State University \\ Raleigh, NC 27695, USA
}

\author{
Stephen Roberts \\ Industrial and Systems Engineering \\ North Carolina State University \\ Raleigh, NC 27695, USA
}

\begin{abstract}
In the event of an outbreak of a highly contagious communicable disease, public health departments often open mass-vaccination or antiviral dispensing clinics to treat the infected population or reduce the further spread of disease. In this research, we have created a simulation of the disease spread process employing a SEIR compartmental model. The model includes employment patterns and separates the population into age groups and spatial location to more accurately describe disease spread behavior. The analysis involves measuring health-related performance as we change the number of days elapsing between clinic days. We open clinics in locations that maximize the infected population coverage subject to budget and resource-related constraints, using a MIP location-allocation model. An example case is provided in the context of an outbreak occurring in Wake County, NC. The simulation is coded in C++, using ILOG Concert Technology to implement the location-allocation model.
\end{abstract}

\section{INTRODUCTION AND MOTIVATION}

Public health emergency planning and response has become a very important topic over the last decade, with the events of 9/11, Anthrax, SARS, Swine Flu (H1N1) and recurring threats of bio-terrorism and infectious disease pandemics. If a public health emergency were to occur, in the form of a novel infectious (communicable) disease outbreak, then no one would have prior immunity to it and we would expect the entire general population to initially be susceptible to the disease. Then, as the susceptible population would come into contact with infectious individuals through normal social interaction. Some of these susceptible individuals then become exposed and eventually infectious themselves.

To treat the ill population as well as reduce the further spread of disease, public health officials can intervene by opening clinics and assigning health resources to these facilities to medicate and/or vaccinate the sick population. In talking to local public health officials, one tool that would be of particular value is a scenario planning tool that could be used to plan for these public health events. Officials would like to know how to respond to a given set of circumstances with various characteristics (infectiousness of the disease, extent of the spread, intervention strategy) with respect to key logistical issues, such as where to locate emergency response clinics and how to allocate key public health and medical resources. One of the aims of this research is to create a framework that could be naturally adapted to such a tool.

The simulation framework detailed in this paper uses the $\mathrm{C}++$ programming language to create a disease spread and facility location simulation that can execute both models simultaneously and seamlessly. The disease spread simulation runs "in the background" and when it is necessary to make facility location decisions, the program externally calls the MIP solver, CPLEX. 


\section{Carr and Roberts}

Countless amounts of research has investigated the use of disease spread models to make public health policy decisions including proper intervention strategies from both cost and efficacy objectives. Compartmental disease spread models have been used for approximately 100 years (Hethcote 2000; Oli et al 2006), while more recently agent-based models have been created to more accurately describe the heterogeneities of social interaction and geography. In this research, a compartmental approach will be taken. However, for ease of definition and to avoid larger computational concerns, the agent-based approach is substituted by a population-based model with added age and geography heterogeneities. Sattenspiel (2009) wrote a book on how varieties of compartmental models have been used to model past pandemics, with special consideration given to geographic models.

Previous literature in the field of industrial engineering and operations research has addressed certain logistical concerns in emergency response planning. Research has been done on triage design for largescale public health emergencies, such as a pandemic, and a number of discrete-event simulations have been created to assist in modeling mass vaccination efforts (Aylwin et al 2006). Ekici (2008) integrated a disease spread model with a model to locate food dispensing facilities. The University of Pittsburgh has recently created a dynamic, discrete event simulation model that integrates GIS to visualize the effects of a public health emergency, while depicting the health status of populations affected as well as the effects of intervention through health care facility location and treatment (Wu et al. 2007). However, this model relies on an expert system, or rule-based, decision model for facility location and resource allocation decisions. Regarding facility location models, Jia et al (2007) addressed the various mathematical models that can be used to respond to large-scale emergencies. In fact, Marianov (2002) discussed a variety of capacitated facility location models used in the public sector.

\section{METHODS AND MODELS}

The following section details the specific models used within the integrated disease spread and facility location simulation for public health emergency response. The formulations of the disease spread and facility location models will be thoroughly explained as well as how they work together to create a useful emergency planning tool.

\subsection{Geographic Disease Spread Model}

A deterministic, compartmental population-based model is used to simulate the spread of a communicable disease throughout a large region, with interactions between smaller regions and age-specific disease parameters. The disease spread process is an adapted version of the SEIR compartmental disease model. Extensions have been made to this disease model to consider the movement of populations into vaccination, antiviral medication, and/or quarantine/self-isolation compartments. Figure 1 shows a pictoral representation of the disease spread process, complete with the potential voluntary actions, interventions, and disease process.

By adding intervention compartments, we can consider the cases when a certain proportion of the population seeks medical treatment or other medical interventions, which change the disease dynamics. However, in the case of antivirals and vaccination, people in these compartments may still be susceptible to disease due to processes such as the natural ability of the immune system to create antibodies and current level of medication in the body able to fight incoming disease. 


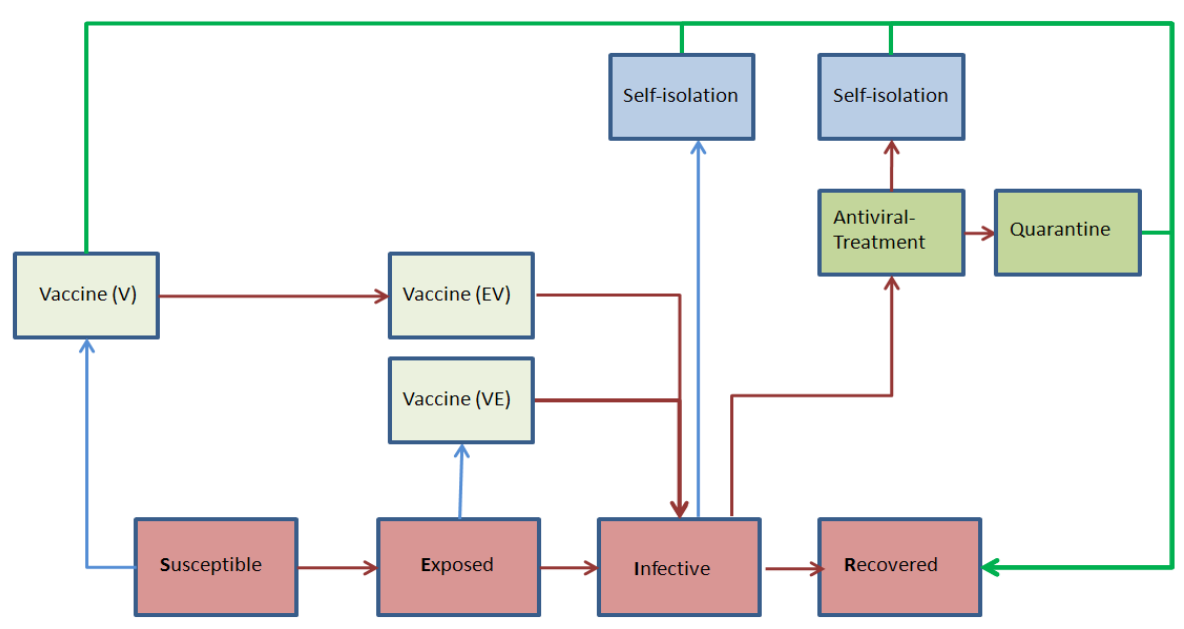

Figure 1: Graphical Representation of the Disease Spread Model.

This model assumes that the entire population is initially susceptible to an upcoming outbreak. From the Susceptible compartment, proportions of the population can voluntarily go to the Vaccine compartment or involuntarily go to the Exposed compartment should they have adequate contact with an infected individual. From the Vaccine compartment, individuals may also become exposed if they come into contact with infected individuals before achieving full immunity from the vaccine. Individuals from the Exposed compartment can also voluntarily go to the Vaccine compartment, as they are not currently aware of being exposed. From the Vaccine (EV) and Vaccine (VE) compartment, individuals proceed to the Infective compartment, where they are now contagious. From this compartment, they may voluntarily go into self-isolation or seek medical treatment with antiviral medication. It is assumed in this model that individuals that voluntarily seek antiviral medication must get this from a medical provider. A proportion of individuals will not voluntarily move anywhere and proceed to the Recovered compartment after an infectious period. From Antiviral Treatment compartment, individuals may voluntarily move into selfisolation, or be deemed too contagious and required to move into quarantine and await full recovery. From all self-isolation compartments, it is assumed that individuals will wait for complete recovery before moving back into the general population, effectively moving them into the Recovered compartment.

The transmission of disease is governed by a force of infection defined as the rate of new infection caused by adequate contacts between infected and still-susceptible individuals. The force of infection can be defined more formally by the following equation:

Force of Infection $=$ Prob(Transmission|Adequate Infectious Contact) * Prob(Adequate Infectious Contact [ Infectious Contact) * Prob(Infectious Contact|Contact) * Number of Contacts Per Unit Time.

The probability that a contact is infected is simply the number of infected individuals from a given contact group divided by the total number of individuals in that group. This assumes that two people with a common contact group share the same probability of coming into contact with an infected individual. For sub-populations with several contact groups, the total force of infectious is the summation of forces of infections over all contact groups. For the working population, it is assumed that the probability that a person is infected is equal to the number of infected individuals in the same age group and region divided by the total number of individuals in that age group and region. 


\section{Carr and Roberts}

\subsubsection{Geographic and Demographic Population Data}

The population of the geographic region simulated is to be broken down into census tract regions. Census tracts are aggregated population regions designated and defined by the U.S. Census Bureau. U.S. Census tract boundary data is publicly available and the corresponding population data can be separated by age, gender, and ethnicity, if desired. By separating the population in both age groups and more specific geographic location, the disease model can more accurately describe the spread of disease geographically, taking into consideration the different levels of interaction among different age groups.

\subsubsection{Employment Data}

The disease spread model also takes into account the employment patterns of those people ages 25-65 (the approximate working population). The model uses data from the On the Map Version 3 online tool created by the U.S. Census Bureau and housed in the Virtual Research Data Center at Cornell University. This data tells us how many people live in one census tract and work in another, including the one in which they live. This information is used to add disease dynamics between geographic regions due to employment patterns. This employment data is used to estimate the number of contacts made between people in different regions. Because people spend approximately half of their active hours at work, their potential for exposure to the communicable disease should be affected by their location of employment.

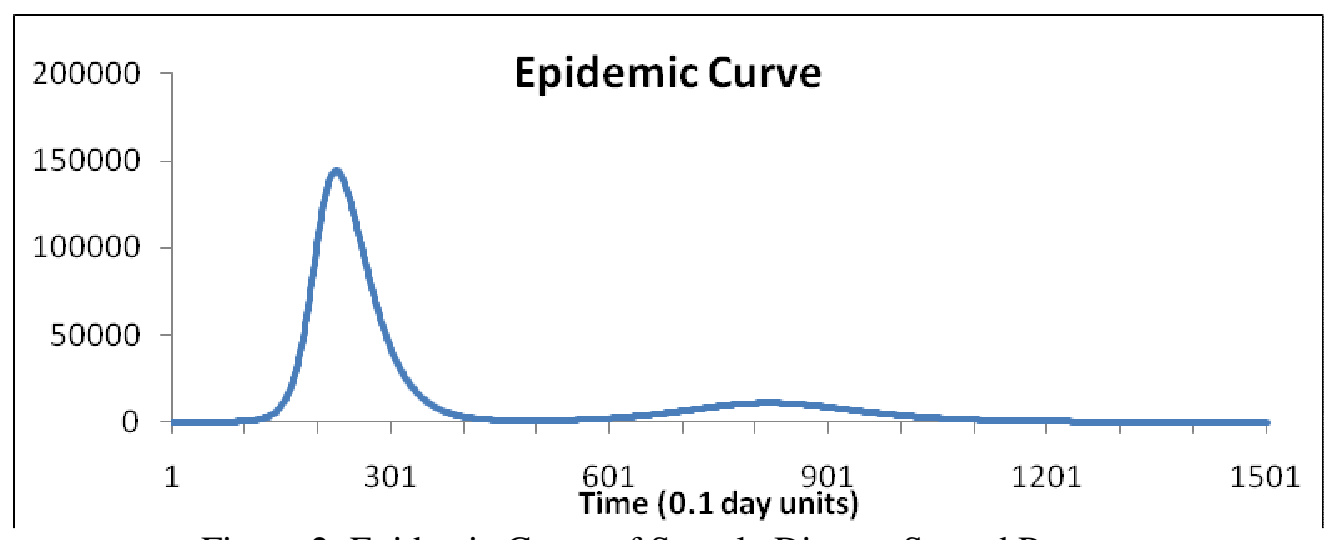

Figure 2: Epidemic Curve of Sample Disease Spread Process

\subsection{Facility Location Model}

The facility location model employed in this research is a capacitated location-allocation model mathematical formulated into a mixed-integer Program. It can be viewed as a centralized decision made by a public health official or decision-maker if a large-scale disease outbreak were to occur. The decisions to be made include: where emergency response facilities will be located, which geographic regions and subpopulations will be assigned to which facilities, and how many healthcare workers will be needed at each facility.

The objective is to serve the largest demand possible subject to the relevant practical constraints. In doing this, we hope to slow the spread of disease by treating a large proportion of the infected population. In reality, the disease may also die out on its own if enough of the infected population are treated or isolated, due to the inherent stochasticity of disease dynamics and social interaction. In order to express practical issues experienced during a public health emergency, constraints are added to the formulation to consider capital budgeting, congestion at the facilities, geographic access to medical services, and availability of medication/supplies. 


\section{Carr and Roberts}

The detailed mathematical formulation is defined below. Constraint (1) expresses the availability of medication. Constraint (2) is a budgetary constraint taking into consideration facilities as well as medical personnel costs. Constraint set (3) is a utilization constraint that controls the amount of people allocated to any one facility by assuming that when both arrivals and service rates are highly random, then the level of congestion (and patient wait times) will not be prohibitively large. Constraint set (4) allocates each geographic region to at most one facility. Constraint set (5) controls the amount of healthcare staff allocated to each facility, which may be of concern when space at the facilities is limited. Constraint set (6) prohibits any population from being assigned to a facility that is too far away. These constraints are intended to ensure a high level of access to care for each region. Of course, if there are some regions relatively far from the closest potential facility, these constraints may need to be relaxed. Finally, Constraint set (7) prohibits populations from being assigned to un-opened facilities. Table 1 is the detailed mathematical formulation while Table 2 outlines the key parameters of the model.

Table 1: Mathematical formulation of the Facility Location Model

$$
\begin{aligned}
& \text { Maximize } \sum_{i} \sum_{j} \lambda_{i} x_{i j} \\
& \text { Subject to } \\
& \sum_{i} \sum_{j} x_{i j} \lambda_{i} \leq V \\
& \sum_{j}\left(f_{j} Y_{j}+s-h_{j}\right) \leq B \\
& \sum x_{i j} \lambda_{i} \leq h_{j} \mu * U \quad \forall j \\
& \sum_{i}^{i} x_{i j} \leq 1 \quad \forall j \\
& h_{j} \leq \Gamma * Y_{j} \quad \forall j \\
& x_{i j} d_{i j} \leq R \quad \forall i_{i j} \\
& x_{i j} \leq Y_{j} \quad \forall i, j
\end{aligned}
$$

\begin{tabular}{|c|c|}
\hline$\lambda_{i}$ & Demand located at population center $i$ \\
\hline$d_{i j}$ & Distance between population center i and facility \\
\hline B & Budget allocated for clinic costs \\
\hline$f_{j}$ & Cost of opening/operating a facility at site j \\
\hline$s$ & Cost of allocating one healthcare worker to facility j \\
\hline$\mu$ & Service rate for healthcare staff \\
\hline$V$ & Number of doses avallable \\
\hline $\mathrm{U}$ & Limit on utilization/traffic intensity at each facility \\
\hline$\Gamma$ & Limit on number of healthcare workers at each facility \\
\hline $\mathrm{R}$ & Service radius for each facility \\
\hline
\end{tabular}

Table 2: Facility Location Model Key Parameters 


\section{Carr and Roberts}

\subsection{Integrated Disease Spread and Facility Location Simulation}

The simulation begins by commencing the disease spread model. In this research, each sub-region pulls a random number. If the random number is below a given threshold $\boldsymbol{X}(0.05$, for example) the given region is assigned one infected individual. Each region performs the same infection initializing process. It is expected that the initial number of infected individuals is the product of threshold value $\boldsymbol{X}$ and the number of regions. If no region is initialized with infection, the region with the largest population can be chosen as the location of the index case of the outbreak.

The rates of change in compartment levels are updated at the rate of a pre-determined step size, which is referred in the model as $z$. With the latest values of the rates of change, the levels are then updated by multiplying the rates by the step size. The disease spread simulation executes in this fashion, updating at every step size. In this way, the simulation is a discretized version of a continuous, dynamic system. However, the step size can be reduced to any value that achieves the desired precision of the simulation.

At fixed intervals, the clinic location model is run using the current infected population as the population input data to the mathematical model. If the objective of the public health decision-makers was to mass-vaccinate, the current susceptible population could be used as input to the location model. The output of the model informs the decision makers which clinics to open, where to assign the infected population, and how many healthcare workers to assign to each opened facility. It is assumed that either all (or a large majority) of the infected population will go to their assigned facility once the clinic is opened. If it is determined that not all of the assigned population will voluntarily seek public health intervention, it is assumed that the same percentage of the total population applies also to each age group.

Within the disease spread simulation, when modeling anti-viral dispensing, the served population moves from the infected compartment to the antiviral treatment compartment. From this compartment, they move to recovered after an average waiting time. However, according to the disease spread model, some of these people may need to be quarantined or may voluntarily move into self-isolation. The strategy employed to make practical use of the Disease Spread and Facility Location Simulation is as follows:

0. Define Response Interval, initialize simulation time $T=0$;

1. Advance Disease Spread Model for one time step

- If simulation time Tis an integer multiple of Response Interval, go to Step 2;

- Else $t=t+1$ and repeat Step 1;

2. Run Location Model

- Input: Set demand equal to infected population for each tract;

- Output: Move assigned populations from infected compartment to antiviral compartment;

- Go to Step 1;

3. Measure health and cost outcomes

- Adjust interval based on objectives and repeat Step 0 as needed;

The simulation may be run for a variety of intervals and objectives to test the cost-effectiveness of opening clinics. As the interval between clinic days is decreased, it is expected that the cost of opening clinics and hiring health care personnel will increase, however the disease may also be better controlled. As the interval increases, the costs of administering aid may decrease, but the disease may spread at a higher rate and infect more total people.

\section{EXPERIMENTAL CASE AND PRELIMINARY RESULTS}

\subsection{Case Example Introduction}

A case example has been used in this research to show the utility of the disease spread and facility location simulation to public health officials in planning for a potential large-scale public health emergency 


\section{Carr and Roberts}

such as an infectious disease outbreak. This case looks at the region of Wake County, North Carolina, as highlighted in Figure 3. This county contains the capital city of Raleigh. The population of Wake County exceeded 750,000 people in 2008.

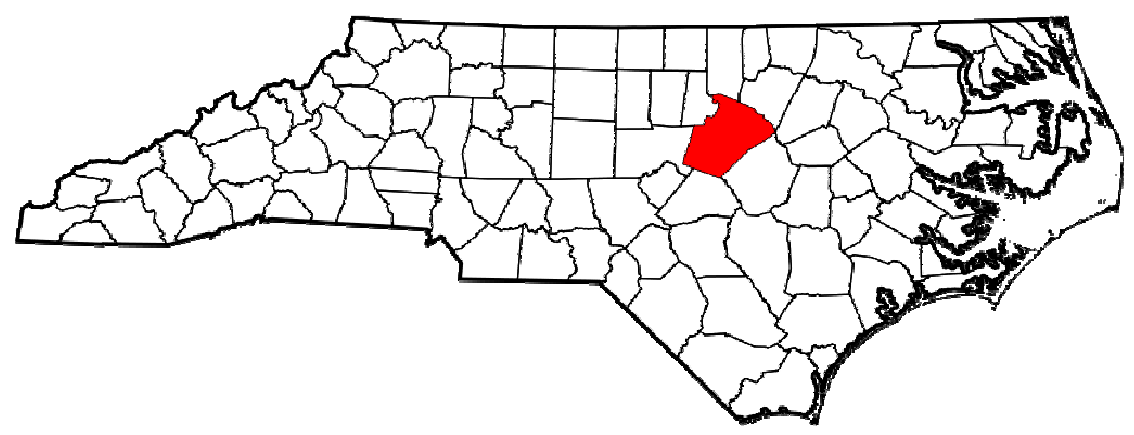

Figure 3: Map of North Carolina highlighting Wake County.

The region is separated by age groups into 105 census tracts. Data was extracted from U.S. Census tract geodata. The tract regions were converted to point data by converting tract boundary polygons to their respective centroids within the ArcGIS software. The facilities available to serve as response clinics are the 103 public elementary schools included on the Wake County School District website. The school locations were also converted to point data using ArcGIS.Figure 4 shows a map of the census tracts of Wake County including the public elementary school locations. Then, using a MATLAB toolbox called MATLOG specialized for logistics applications, distance between schools and regions and among schools was calculated using greatest circle distance and adjusted for local road distance by multiplying by a factor of 1.2 .

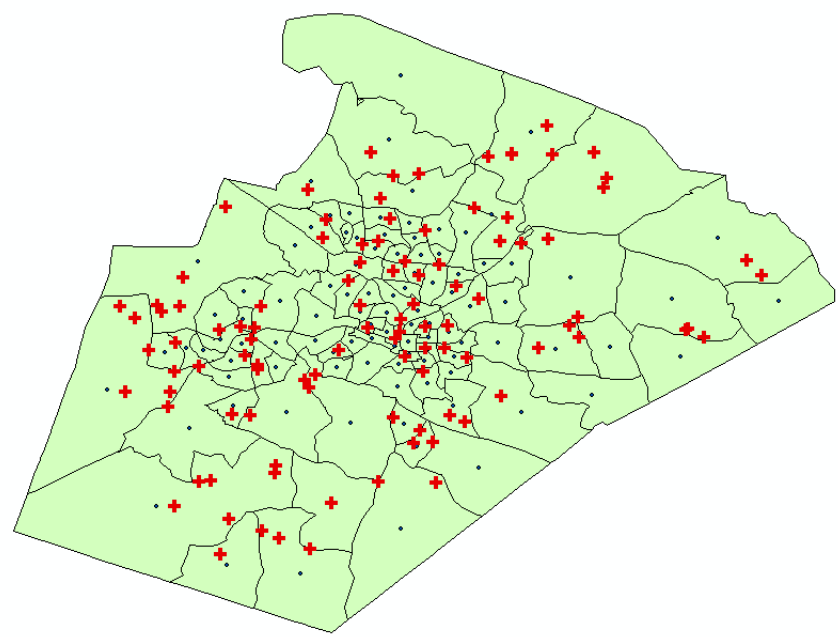

Figure 4: Map of Wake County, NC, highlighting census tracts, their centroids, and public elementary school locations.

\subsection{Case Parameters}

After the setting for an experimental case example is determined, it is then necessary to identify the specific model parameters used within the models, taking into consideration that any results to a model represent results conditioned upon the inputs of the model. For the purposes of this preliminary research, it was determined that the values for many important parameters would be estimated by the authors and the included results would be intended first and foremost for illustrative purposes. For an experimental 


\section{Carr and Roberts}

case, the disease dynamics drew upon previous estimates from epidemiology and is intended to mimic a highly-contagious communicable disease similar to a novel strain of $\mathrm{H} 1 \mathrm{~N} 1$, which has considerable value given the events of the last year.

The facility location model takes parameters that consider the number and availability of public health and medical personnel. It is assumed that these workers are paid for their services during response clinic dates. Budget and medication availability are initial estimates made by the authors and are intended to assist in illustrating the value of the simulation.

The simulation also has the capacity to change the availability of resources dynamically with time, if estimates of these changes are available. For example, public health departments may be able to raise funds and acquire grants once an outbreak is discovered. In addition, the number of doses available initially is drawn from a stockpile, but may also grow in size over time as manufacturers deliver more to providers and public health departments. Therefore, the simulation will begin with fixed availability and change the level of these resources over time. Key location model parameters are outlined in Table 3.

Table 3: Location Model Parameter Values.

\begin{tabular}{|c|c|}
\hline Parameter & Case Example Value \\
\hline $\mathbf{B}$ & $\$ 75,000$ \\
\hline$f_{j}$ & $\$ 2,000$ \\
\hline$s$ & $\$ 200$ \\
\hline$\mu$ & 12 people per hour \\
\hline$V$ & 35,000 stockpile $+10-20,000$ per week \\
\hline $\mathrm{U}$ & $0.8(80 \%)$ \\
\hline$\Gamma$ & 20 \\
\hline $\mathbf{R}$ & 5 miles \\
\hline
\end{tabular}

The disease spread model also needs to be initialized with disease-specific parameters, as well as the level of interactions among age groups. The choice of parameters such as infectious period, latent period, vaccination period, antiviral period, treatment period, and quarantine period have been approximated using information from the Control of Communicable Diseases Manual, $18^{\text {th }} \mathrm{Ed}$. and discussions with local contacts with medical and public health epidemiology expertise. Parameters corresponding to voluntary behavior such as seeking vaccination and self-isolation have been estimated by the modelers and should be further investigated and validated as part of this research. These parameters are outlined in Table 4.

Table 4: Disease Spread Parameter Values

\begin{tabular}{|c|c|}
\hline Parameter & Case Example Value \\
\hline Infectious Period (INF) & 5 days \\
\hline Latent Period (LAT) & 3 days \\
\hline Vaccination Period (VAC) & 14 days \\
\hline Antiviral Period (AV) & 3 days \\
\hline Treatment Period (TR) & 3 days \\
\hline Quarantine Period (Q) & 7 days \\
\hline $\mathrm{P}_{\text {eve }}$ & 0.15 \\
\hline $\mathrm{P}_{\mathrm{iq}}$ & 0.1 \\
\hline $\mathrm{P}_{\mathrm{afq}}$ & 0.15 \\
\hline $\mathrm{P}_{\mathrm{aq}}$ & 0.1 \\
\hline $\mathrm{P}_{\mathrm{sv}}$ & 0.15 \\
\hline $\mathrm{P}_{\mathrm{ia}}$ & 0.2 \\
\hline
\end{tabular}




\section{Carr and Roberts}

An important issue to consider here is that such a disease spread model has not been proposed or detailed in previous research, and could only be validated by fitting the model parameters to past disease data. In the absence of historical data and with a model containing a large number of parameters, we have chosen to calibrate the disease spread parameters to achieve an approximate peak infectivity of $20 \%$, which represents a significant outbreak event. Peak infectivity is a health-related measure often reported after outbreak events.

The age-specific parameters are preliminary estimates that imply a higher level of interactions leading to infection among the very young (ages $0-4$ ) who have less hygienic behavior and among the working ages, who have larger social networks. Sample age groups easily defined using U.S. Census data that reasonably categorize ages by similar social network sizes and levels of interaction are outlined in Table 4. The parameters used to describe age-specific parameters are also shown in Table 4, which shows one-way interactions and shows how infection spreads from one age group to another in terms of contact rates. The values within the From-To chart should be interpreted as an aggregate measure of how many new infections will be created from the interaction between groups of susceptible and infected individuals, and includes the degree of infectivity of the group's daily behavior as well as the number of contacts between two groups, see Table 5.

Table 5: Age Groups and Age-Specific Contact Rates Used in the Disease Spread Model.

\begin{tabular}{|c|}
\hline Age Groups \\
\hline 0-4 years old \\
\hline 5-13 years old \\
\hline $14-24$ years old \\
\hline $25-65$ years old \\
\hline $65+$ years old \\
\hline
\end{tabular}

\begin{tabular}{|c|c|c|c|c|c|}
\hline From-To & $0-4$ & $5-13$ & $14-24$ & $25-65$ & $65+$ \\
\hline $0-4$ & 1 & 0.5 & & 0.1 & \\
\hline $5-13$ & 0.5 & 0.5 & & & \\
\hline $14-24$ & & & 0.5 & & \\
\hline $25-65$ & 0.5 & 0.5 & & $\begin{array}{c}1.0 \mathrm{~N}^{*} \mathrm{E}^{*} \\
0.1 \mathrm{E}^{*}\end{array}$ & \\
\hline $65+$ & & & & & 0.5 \\
\hline $\begin{array}{l}\text { "N-E: Non-Employment Contact Rate } \\
\text { "E: Employment Contact Rate }\end{array}$ \\
\hline
\end{tabular}

\subsection{Case Example Results}

For the illustrative case example, the disease is allowed to progress for 10 days. This is intended to represent the time it would take for the public health community to notice that an outbreak has started and plan for the first clinic date. At day 7, the first set of clinics is opened and the infectious population is served. Thereafter, clinics will be opened every 7 days until the outbreak has been stopped. The facility locations were solved using ILOG Concert Technologies C++ capabilities. Each iteration allowed for a $5 \%$ optimality gap with an additional 5 minute limit on computation time for larger, more difficult problem instances.

From Figure 10, we see the first set of clinics slightly lowers the epidemic curve. However, due to the infection still persisting with an abundance of susceptible people in the population, the epidemic rises dramatically to day 24 , the second clinic date. At this point, it is believed that the epidemic has nearly reached its peak, but the second clinic serves a large amount of infected individuals. Clinic dates 3,4, and 5 also serve some infected individuals and assist in stopping the epidemic.

From Figure 5, we also see that there is a second outbreak in both cases that occur after the first outbreak has nearly been extinguished. This is believed to be largely due to a certain proportion of initially susceptible individuals voluntarily seeking self-isolation. After the first outbreak is over and those in self isolation return to their social settings, as long as there is still one infected person, the outbreak has the ability to spread again. 


\section{Carr and Roberts}

Table 6 shows the key health measures of the outbreak, including peak, cumulative, and average infectivity. Peak infectivity is a measure of the maximum number of people infected at one time. After the peak of the epidemic occurs, people leave the infected compartment faster than susceptible people become infected. Cumulative infectivity measures the sum of the number of people infected throughout the entire outbreak and approximates the area under the epidemic curve. Cumulative infectivity is a way to compare the total cumulative effect of the outbreak for different scenarios, as the peak infectivity is not always a fair comparison. Average infectivity divides the cumulative infectivity by the duration of the outbreak.

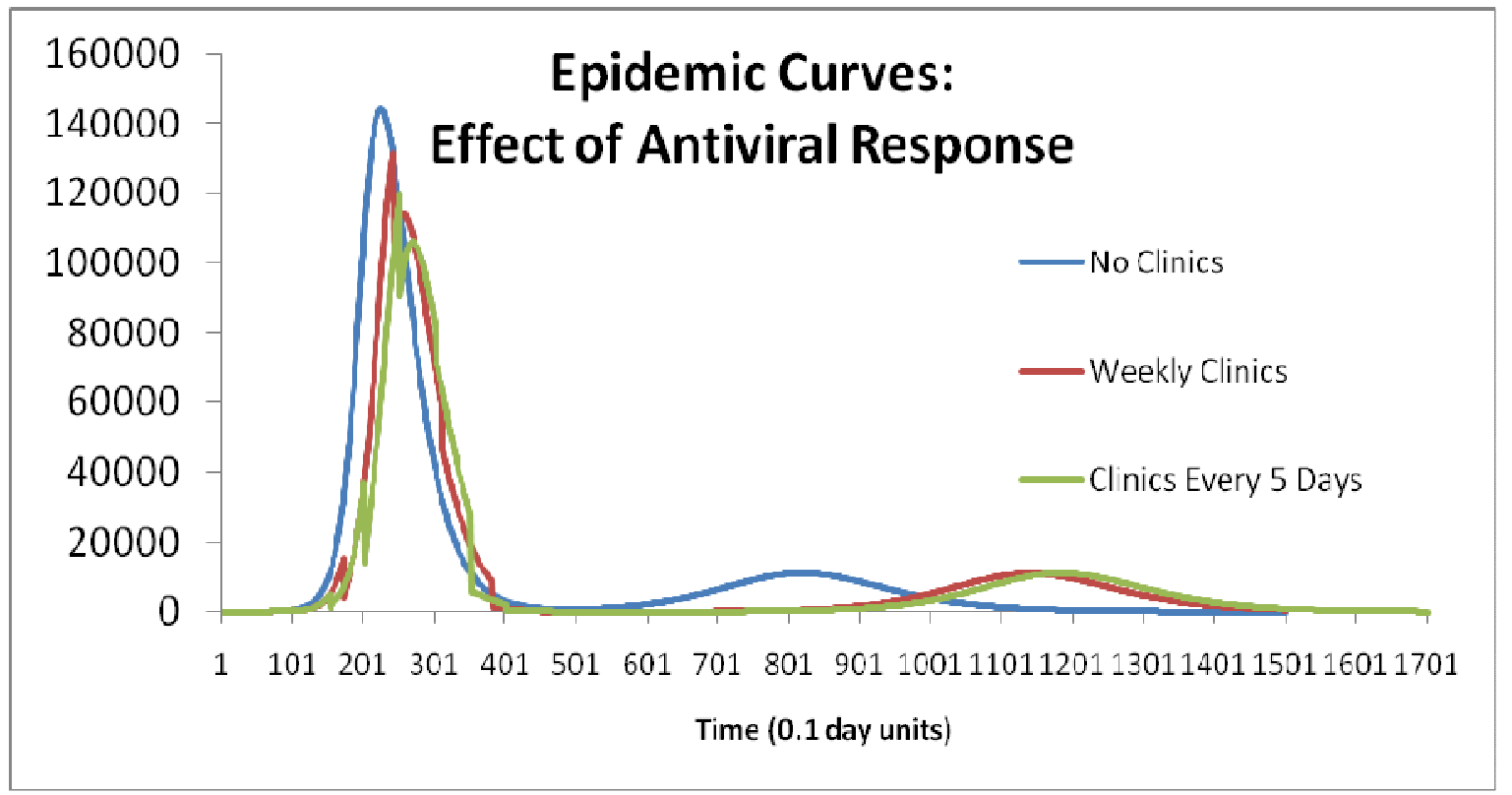

Figure 5: Comparison of Infection Dynamics over Time

Table 6: Key Health-related Measures from Case Example

\begin{tabular}{|c|c|c|c|}
\hline & $\begin{array}{c}\text { Peak } \\
\text { Infectivity } \\
\text { (People) }\end{array}$ & $\begin{array}{c}\text { Cumulative } \\
\text { Infectivity } \\
\text { (People*Days) }\end{array}$ & $\begin{array}{c}\text { Average } \\
\text { Infectivity } \\
\text { (People) }\end{array}$ \\
\hline $\begin{array}{c}\text { No } \\
\text { Clinics }\end{array}$ & $\begin{array}{c}144,119 \\
\text { @ Day 23 }\end{array}$ & $1,802,427$ & 12,016 \\
\hline $\begin{array}{c}\text { Weekly } \\
\text { Clinic }\end{array}$ & $\begin{array}{c}131,444 \\
\text { @ Day 24 }\end{array}$ & $1,599,645$ & 10,664 \\
\hline $\begin{array}{c}\text { Clinic Every } \\
\text { 5 Days }\end{array}$ & $\begin{array}{c}119,783 \\
\text { @ Day 25 }\end{array}$ & $1,512,859$ & 10,086 \\
\hline
\end{tabular}

Table 7 highlights the response activities and requirements for a weekly intervention strategy. Comparing the total number of doses with the peak infectivity, we see that although greater than 54,000 doses were provided, the peak infection decreased a bit more than 12,000. Comparing the total doses and/or cost with the change in infectivity measures also allows for cost-effectiveness or efficiency analyses. 


\section{Carr and Roberts}

Table 7: Facility and Resource Requirements for Weekly Response Clinics

\begin{tabular}{|c|c|c|c|c|}
\hline & $\begin{array}{c}\text { Total Number } \\
\text { of Facilities }\end{array}$ & $\begin{array}{c}\text { Total Number } \\
\text { of Healthcare } \\
\text { Staff Required }\end{array}$ & $\begin{array}{c}\text { Total } \\
\text { Doses }\end{array}$ & Cost \\
\hline Clinic Day 1 & 21 & 21 & 608 & $\$ 46,200$ \\
\hline Clinic Day 2 & 12 & 114 & 13,802 & $\$ 46,800$ \\
\hline Clinic Day 3 & 13 & 189 & 19,387 & $\$ 63,800$ \\
\hline Clinic Day 4 & 6 & 101 & 11,613 & $\$ 32,200$ \\
\hline Clinic Day 5 & 27 & 91 & 8,874 & $\$ 72,200$ \\
\hline TOTAL & 79 & 516 & 54,284 & $\$ 261,200$ \\
\hline
\end{tabular}

Table 8 highlights the response activities and requirements for a weekly intervention strategy. Comparing the total number of doses with the peak infectivity, we see that although greater than 54,000 doses were provided, the peak infection decreased a bit more than 12,000. Comparing the total doses and/or cost with the change in infectivity measures also allows for cost-effectiveness or efficiency analyses.

Table 8: Facility and Resource Requirements for 5-Day Interval Response Clinics

\begin{tabular}{|c|c|c|c|c|}
\hline & $\begin{array}{c}\text { Total Number } \\
\text { of Facilities }\end{array}$ & $\begin{array}{c}\text { Total Number } \\
\text { of Healthcare } \\
\text { Staff Required }\end{array}$ & $\begin{array}{c}\text { Total } \\
\text { Doses }\end{array}$ & Cost \\
\hline Clinic Day 1 & 21 & 21 & 608 & $\$ 46,200$ \\
\hline Clinic Day 2 & 19 & 49 & 4,415 & $\$ 47,800$ \\
\hline Clinic Day 3 & 13 & 245 & 28,053 & $\$ 75,000$ \\
\hline Clinic Day 4 & 13 & 240 & 26,915 & $\$ 74,000$ \\
\hline Clinic Day 5 & 4 & 80 & 9,200 & $\$ 24,000$ \\
\hline Clinic Day 6 & 14 & 235 & 26,663 & $\$ 75,000$ \\
\hline Clinic Day 7 & 30 & 36 & 2,211 & $\$ 67,200$ \\
\hline TOTAL & 114 & 906 & 98,065 & $\$ 409,200$ \\
\hline
\end{tabular}

Figure 6 shows a GIS representation of the disease spread and facility location decisions over time for 5-day clinics. GIS is a useful tool in visualizing the effects of intervention strategies, especially location decisions that contain little context when provided to public health practitioners in a list format. 


\section{Carr and Roberts}

DAY 15

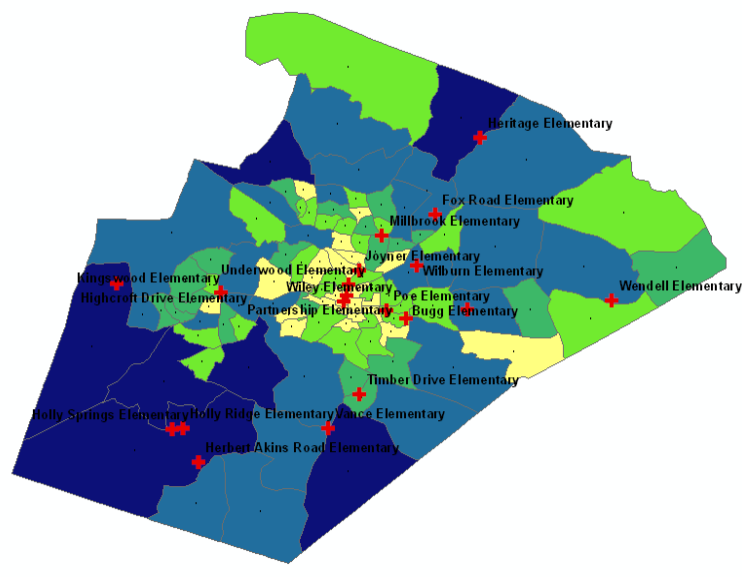

DAY 25

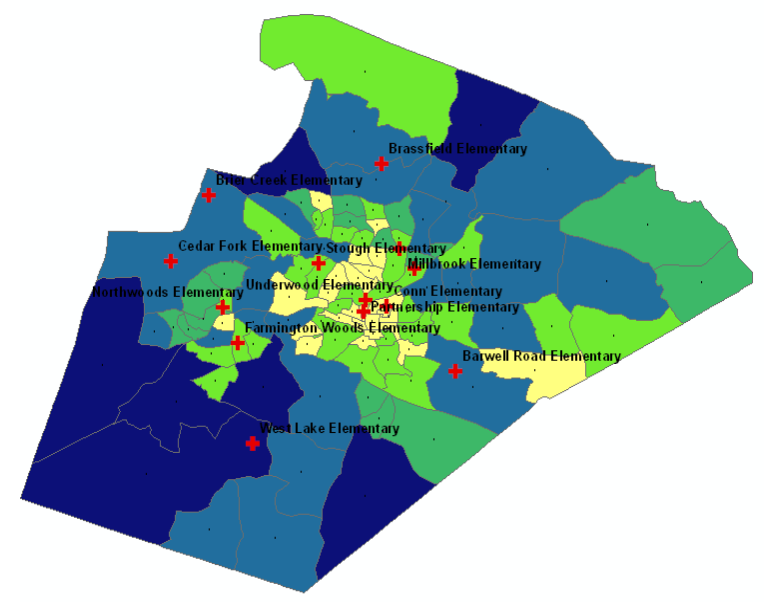

DAY 35

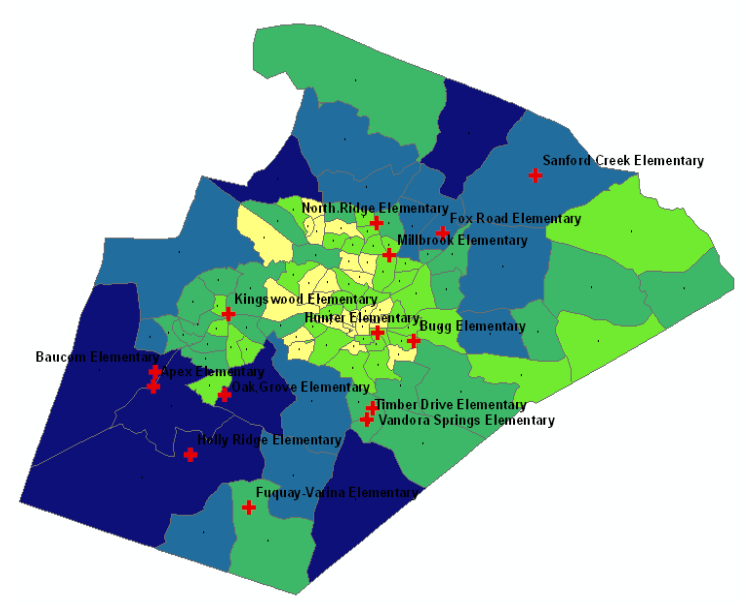

DAY 20

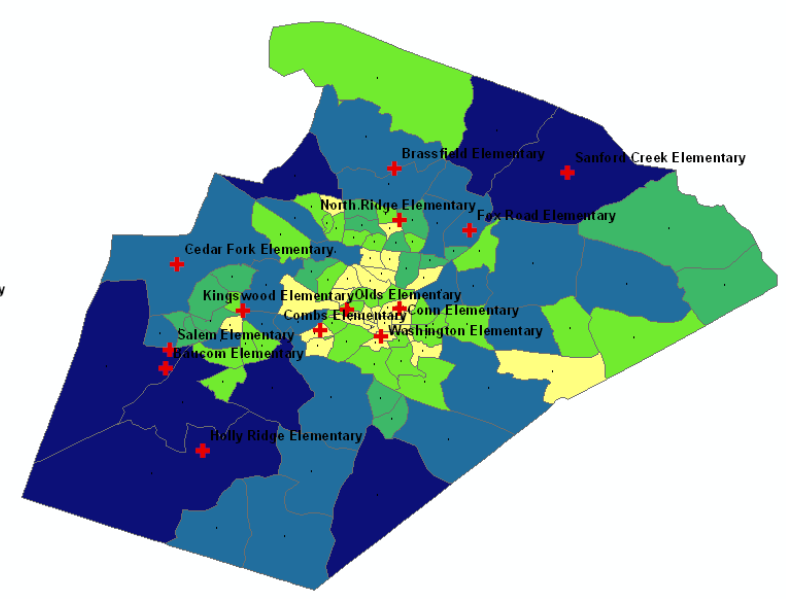

DAY 30

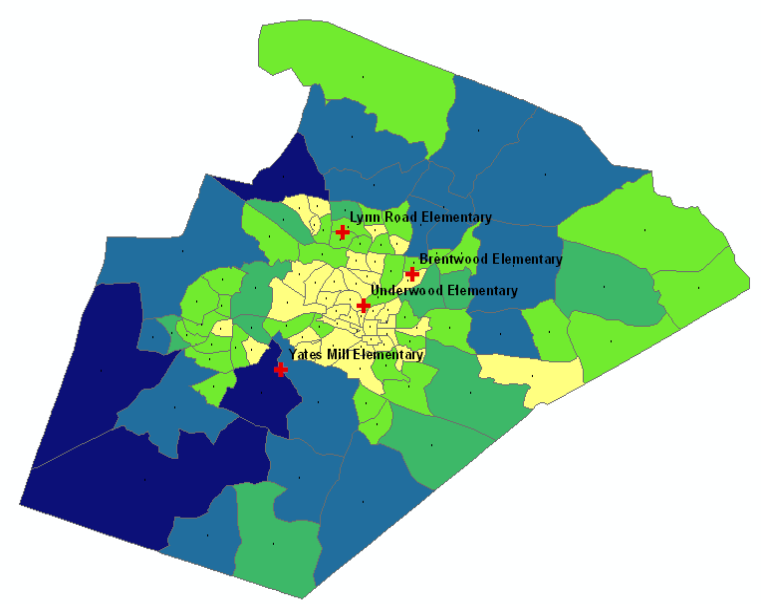

DAY 40

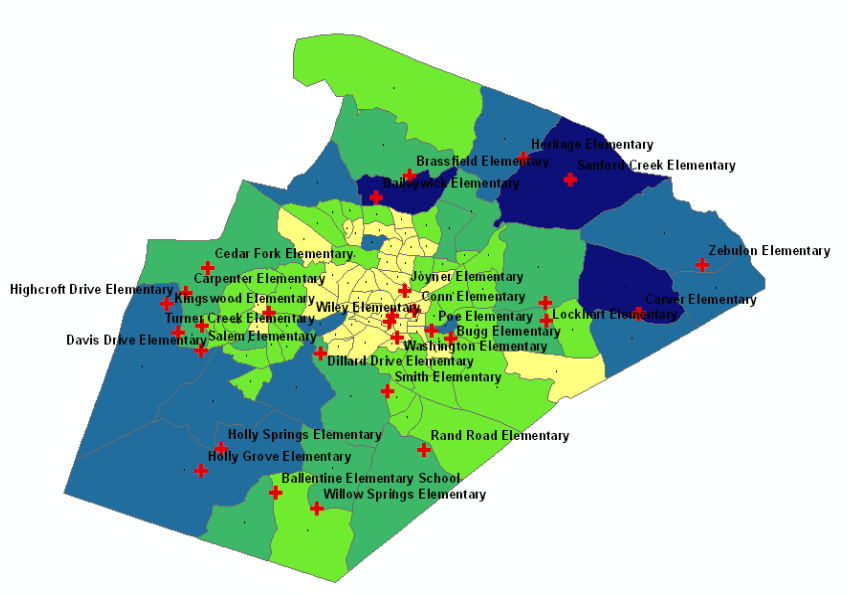

Figure 6: GIS Snapshots of Disease Spread and Facility Locations for 5-Day Clinics 


\section{Carr and Roberts}

A large amount of this research is novel in methodology and its detailed components. This causes concern in model validation as there is little existing data to compare with the results of the model. The disease spread model within this research is intended to create hypothetical large-scale public health events to be used by public health officials to plan for true events, should they happen. The majority of past research in disease spread dynamics have attempted to fit homogeneous SIR, SEIR, and SEIRS models to past events. Here, age-specific and geographic disease spread behavior is intended to give the disease spread more realism, as we know that very few cases are truly homogeneous. In this regard, any disease spread model that is believed to validly represent true disease dynamics within a population would be useful within the proposed framework. Similarly, choosing realistic simulation parameters such as budget and the availability of medication requires data and the modeler should strive to seek as much input from public health officials as possible.

Key results from the research presented here show how response in the form of antiviral medication of infected individuals helps to lessen the extent of an infectious disease outbreak. We have seen that although the number of doses administered does not have a one-to-one correspondence to the decrease in peak infectivity, the number of doses also contributes to increased productivity by shortening the illness of those infected. Future work involves in-depth sensitivity analysis on both the disease spread and resource-related parameters to be able to measure the effect of contact patterns, costs, resource availability, and timing of clinic dates on the size and duration of epidemics. However, it is clear that such a tool has the ability to aid public health organizations plan and respond to large-scale public health emergencies including epidemic outbreaks. The ultimate goal of this research will be to create a valid scenario planning tool for these public health entities. We believe the framework of this research gives the ability for decision makers to compare countless scenarios in a rapid, yet informational manner.

The choice of potential facilities also has an effect on the performance of the facility location model. As the number of potential facilities increases, the feasible region of the problem also increases. This leads to many more solutions that must be investigated within any solution methodology, including branch and bound. If the choice of potential facility locations can be significantly decreased (perhaps by considering practical issues such as road access, parking availability, and size of school) then the optimal choice of facilities would be found with much less computational effort.

This research has the potential for several extensions. The disease spread model can be extended in many directions, including adapted to an agent-based framework. Meta-heuristics such as genetic algorithm can also be applied to the integrated disease spread model in the hopes to optimize the timing of opening clinics and other practical issues such as the best use of allocated funds.

\section{ACKNOWLEDGMENTS}

This research is a part of the North Carolina Preparedness and Emergency Response Research Center (NCPERRC) and was supported by CDC Grant 1PO1 TP 000296-01.

\section{REFERENCES}

Aylwin, C. 2006. Reduction in critical mortality in urban mass casualty incidents: analysis of triage, surge, and resource use after the London bombings on July 7, 2005. Lancer 368:2219-2225.

Daskin, M. and L. Dean. 2004. Location of Health Care Facilities. Operations Research and Health Care: A Handbook of Methods and Applications. Brandeau, M. et al, eds. Kluwer, Boston

Ekici, A., P. Keskinocak, and J. Swann. 2008. Pandemic Influenza Response. In Proceedings of the 2008 Winter Simulation Conference, eds. S. J. Mason, R. R. Hill, L. Mönch, O. Rose, T. Jefferson, J. W. Fowler. Piscataway, New Jersey: Institute of Electrical and Electronics Engineers, Inc. 


\section{Carr and Roberts}

Heymann, D., eds. 2004. Control of Communicable Diseases Manual, $18^{\text {th }}$ Ed. American Public Health Association, Washington, D.C.

Hethcote, H.W. 2000. The Mathematics of Infectious Disease. SIAM Review. 42(4): 599-653.

Jia, H., F. Ordonez, and M. Dessouky. 2007. A modeling framework for facility location of medical services for large-scale emergencies. IIE Transactions 39 (1):41-55.

Longini, I. M., M. E. Halloran, A. Nizam, and Y. Yang. 2004. Containing Pandemic Influenza with Antiviral Agents. American Journal of Epidemiology 159: 623-633.

Marianov, V. and D. Serra. 2002. Location Problems in the public sector. Facility Location: Applications and Theory. Drezner, Z. and H. Hamacher, eds. Springer, Berlin.

Oli, M. K., M. Venkataraman, P. Klein, L. Wendland, and M. Brown. 2006. Population dynamics of infectious diseases: A discrete time model. Ecological Modelling 198 (1-2):183-194.

Sattenspiel, L. The Geographic Spread of Infectious Diseases Princeton Press, New Jersey: 2009.

Zhang,Y., O. Berman, and V. Verter. 2009. Incorporating congestion in preventive healthcare facility network design. European Journal of Operational Research 198( 3): 922-935.

Wu, S., L. Shuman, B. Bidanda, M. Kelley, B. Lawson, K. Sochats, and C. Balaban.2007. System implementation issues of Dynamic Discrete Disaster Decision Simulation System (D4S2) In Proceedings of the 2007 Winter Simulation Conference, eds. S. G. Henderson, B. Biller, M.-H Hsieh, J. Shortle, J. D. Tew, and R. R. Barton. Piscataway, New Jersey: Institute of Electrical and Electronics Engineers, Inc.

\section{AUTHOR BIOGRAPHIES}

SEAN CARR is a Ph.D. student in the Edward P. Fitts Department of Industrial and Systems Engineering at North Carolina State University. His email address is <smcarr2@ncsu . edu $>$.

STEPHEN ROBERTS is the A. Doug Allison Distinguished Professor in Health Systems Engineering in the Edward P. Fitts Department of Industrial and Systems Engineering at North Carolina State University. His e-mail address is <robertsencsu. edu> 